\section{Hospital Humanitario Pablo Jaramillo. Pediatría. Cuenca - Ecuador}

\section{Artículo Original Original Article}

DOI: https://doi.org/10.18537/RFCM.38.03.05

Correspondencia:

wilblavi@hotmail.com

\section{Dirección:}

Benjamín Carrión y Benigno Vela

Código Postal:

010105

\section{Teléfono:}

0993879036

Cuenca - Ecuador

\title{
Anemia y estado nutricional en menores de 5 años. Hospital Pablo Jaramillo Crespo, Cuenca-Ecuador.
}

Anemia and nutritional status in children under 5 years of age. "Hospital Pablo Jaramillo Crespo", Cuenca-Ecuador

Blacio Vidal Willie Jack ${ }^{1}$

\section{RESUMEN}

La anemia representa una enfermedad con una elevada prevalencia que está ligada a situaciones precarias que viven los infantes.

Objetivos: determinar la frecuencia de la anemia y establecer su relación con el estado nutricional en menores entre 6 y 59 meses, ingresados al Hospital Pablo Jaramillo Crespo. Cuenca-Ecuador, 2017.

Metodología: estudio retrospectivo que estableció la frecuencia de la anemia en menores entre 6 y 59 meses hospitalizados durante enero a diciembre de 2017, determinando el vínculo con las condiciones nutritivas del involucrado. El análisis se realizó mediante el programa Epi-info y la prueba de asociación utilizada fue Chi-cuadrado de Pearson, admitiéndose una $p \leq 0.05$ para establecer significancia estadística.

Resultados: el $52.6 \%$ de los niños fueron del género masculino y $47.4 \%$ femenino, predominando los de edad preescolar (51.9\%). La frecuencia de anemia fue del $39.6 \%$, de los niños con anemia el $6 \%$ tuvieron desnutrición moderada a grave existiendo significancia estadística. Los niños con anemia fueron del sexo femenino (51.3\%) y presentaron desnutrición en el $23.9 \%$, mientras que de aquellos sin esta enfermedad el $55.2 \%$ perteneció al masculino y el $18.6 \%$ estaba desnutrido.

Conclusión: seencontró que la anemia tiene relación estadísticamente significativa con la desnutrición..

Palabras clave: trastornos de la nutrición del lactante, trastornos de la nutrición del niño, anemias nutricionales, sobrepeso, obesidad. 


\section{ABSTRACT}

Anemia represents a disease with a high prevalence that is connected to the precarious situations that infants experience.

Objectives: to determine the frequency of anemia and establish its relationship with the nutritional status in minors between 6 and 59 months admitted to the "Hospital Pablo Jaramillo Crespo". Cuenca-Ecuador, 2017.

Methodology: it is a retrospective study that established the frequency of anemia in children between 6 and 59 months hospitalized during January to December 2017, determining the link with the nutritional conditions of the patient. The analysis was carried out using the Epi-info program and the association test used was Pearson's Chi-square, admitting a $p \leq 0.05$ to establish statistical significance.

Results: $52.6 \%$ of the children were male and $47.4 \%$ female, pre-school age children predominating (51.9\%). The frequency of anemia was $39.6 \%$, of the children with anemia $6 \%$ had moderate to severe malnutrition, existing statistical significance. The children with anemia were female (51.3\%) and 23.9\% presented malnutrition, while children without this disease $55.2 \%$ belonged to the male and $18.6 \%$ were malnourished.

Conclusion: it was found that anemia has a statistically, significant relationship with malnutrition.

Key words: child nutrition disorders, infant nutrition disorders, nutritional anemias, overweight, obesity.

\section{INTRODUCCIÓN}

Cerca del $25.7 \%$ de los niños en etapa preescolar sufren de anemia, con una prevalencia mayor para el sexo masculino en comparación al femenino (26.8\% Vs. 24.6\%), llegando a un 62\% para aquellos menores a 1 año, estas cifras van en conformidad con la Encuesta Nacional de Salud y Nutrición Ecuador (ENSANUT-ECU 2012-2018). Sin embargo, la desnutrición per se a nivel mundial se ha disminuido notablemente desde 1986, esto se ha dado también por el aumento del sobrepeso y la obesidad' ${ }^{1}$.

Aun cuando el estado nutricional involucra un equilibrio entre los requerimientos alimenticios y el consumo habitual de una persona, en el caso de los niños con 5 años o menos esta nivelación quizás se vea afectada por varias razones, que acarrean consecuencias hacia la velocidad del crecimiento de ellos, deterioro de las defensas inmunológicas, actividad física y el desarrollo normal del infante, por lo cual adquiere relevancia que exista libre acceso, disponibilidad al consumo de insumos nutricionales que conlleven al cumplimiento de las necesidades esenciales para evitar la malnutrición².

Por otro lado, la anemia ferropénica condiciona la disminución de las habilidades y destrezas que tiene un niño para aprender y limita sus posibilidades a largo plazo, esto puede llegar incluso a repercutir en la evolución de la población. No obstante, la Organización Mundial de la Salud (OMS) expone que esta patología puede afectar a un aproximado de 800 millones de mujeres y niños, igualmente, para el año 2011 había 273 millones de infantes con edades por debajo de los 5 años con anemia y casi la mitad tenía ferropenia ${ }^{3}$.

En vista de la prevalencia paulatina hasta el año 2008, la anemia se ha tenido presente como un problema de salud en todo el mundo, bajo esta premisa, la OMS otorgó datos de 192 miembros de esta organización que exponen una frecuencia global de $25.4 \%$ para los niños 
que se encuentran en el período escolar y para los que están en preescolar de un $47.4 \%$, donde en el segundo grupo la prevalencia fue variando con el país y hubo mayor afectación en las zonas de África, la India y América del Sur ${ }^{4}$.

Bajo estas consideraciones, el estado nutricional se ha visto afectado por las altas cifras de sobrepeso y obesidad como ya fue manifestado por la OMS, de hecho, a nivel global el número de niños menores a 5 años que tienen estas comorbilidades se ha incrementado de 32 millones en 1990 a 41 millones en 2016. En el caso de África, la OMS expuso que estos datos se han elevado de 4 a 9 millones durante el mismo período de tiempo, pero, afortunadamente, la cantidad de desnutrición se ha reducido ${ }^{5}$.

En términos generales, la anemia infantil se considera un factor de riesgo que implica diversas consecuencias que no distinguen el origen de ésta, mostrándose a corto y a largo plazo siendo crónica o aguda. En el caso de la primera, el niño puede presentar fatiga, taquicardia y disnea donde el peor escenario es cuando hay una redistribución de la sangre tanto hacia el cerebro como al corazón, entonces se da una reducción de la cantidad de oxígeno en otros órganos que no son tan importantes pero que, por ejemplo, puede generar una isquemia intestinal con translocación bacteriana que eventualmente culmina en la falla múltiple de los órganos vitales o importantes ${ }^{6}$.

Por su parte, la anemia crónica tiene efectos que comprometen el adecuado desarrollo del infante haciéndolo propenso a una salud irregular durante la adultez, estas consecuencias son: perturbaciones en el desarrollo cognitivo, retraso del desarrollo ponderal, problemas cardíacos y enfermedades óseas ${ }^{7,8}$.
Para los niños, esta enfermedad se traduce en un deficiente desarrollo tanto motriz como cognitivo, bajo rendimiento escolar del mismo modo que una creciente morbilidad y mortalidad ${ }^{9}$. A nivel social, hay indicios fuertes de que la anemia tiene repercusiones en la eficiencia tanto socioeconómica como productiva de un país ${ }^{10}$, por lo tanto, tomando en cuenta todo lo dicho, el objetivo de este trabajo investigativo es poder determinar el vínculo entre la anemia con el estado nutricional de los niños entre 6 y 59 meses.

\section{METODOLOGÍA}

Se realizó un estudio analítico de prevalencia en el Hospital Humanitario Especializado Pablo Jaramillo Crespo que se encuentra en la ciudad de Cuenca, Azuay en el período entre enero diciembre del año 2017, donde se consideró un universo compuesto por 285 pacientes con un rango de edad entre los 6 y 59 meses, a quienes se les hizo lo siguiente:

Utilización de todos los datos conseguidos a partir de la biometría hemática completa, pues habitualmente se les solicita a todas las personas admitidas para obtener la información de los hematocritos y hemoglobina, así como si tiene anemia.

A partir de este reporte sobre la hemoglobina, se hizo una corrección de los valores tomando en consideración la altura de la ciudad de Cuenca (2640 m.s.n.m) que implicó un ajuste por remoción de 1.3 a los valores de acuerdo con la Atención Integrada a Enfermedades Prevalentes de la Infancia (AIEPI) 2017.

A partir de las historias clínicas se recopilaron todas las medidas antropométricas (estatura y peso), estableciendo una relación entre ambos parámetros para obtener una clasificación ajustada al estado nutricional, siendo estas, 
de acuerdo a los valores $Z$ de las curvas de la OMS: desnutrición grave, moderada o leve, eutrófico y sobrenutrición que puede implicar obesidad mórbida, obesidad y sobrepeso.

Criterios de inclusión: historias clínicas de infantes con un rango etario de 6 a 59 meses que fueron admitidos de enero a diciembre de 2017.

Criterios de exclusión: infantes que tengan algún indicio de otra patología crónica.

El análisis de datos obtenidos fue explicado mediante tablas con medidas de frecuencia y tendencia central, empleando el programa Epi info. Además, se evaluaron las posibles asociaciones entre las variables con el Chi cuadrado aceptando un $\mathrm{p}<0.05$ como estadísticamente significativa.

\section{RESULTADOS}

Se evidenció que el 52.6\% fueron niños y el $47.4 \%$ niñas. Con respecto al grupo etario, el $51.9 \%$ fueron preescolares, $29.8 \%$ lactantes mayores y lactantes menores el 18.2\%, la prevalencia de anemia en toda la población fue del $39.6 \%$, según el estado nutricional el $65.3 \%$ fueron eutróficos, $20.7 \%$ desnutridos y $14 \%$ sobrepeso-obeso (Tabla $N^{\circ} 1$ ).

De los pacientes con anemia el 6.0\% fueron desnutridos moderados-graves versus el $1.4 \%$ de este grupo que no presentó anemia, demostrándose un comportamiento estadísticamente significativo (Chi cuadrado: $4.00 p=0.005)($ Tabla N²).

En la población total, de todos los evaluados que sonanémicos el género masculino representó el $48.7 \%$ y el femenino el $51.3 \%$, comportamiento no estadísticamente significativo (Tabla Nㅜㄱ).

Se observa que, de toda la población, en aquellos con anemia la desnutrición se presentó en un 23.9\%. Por otro lado, para los que no tuvieron esta enfermedad, la desnutrición se registró en el $18.6 \%$ de estos, comportamiento no estadísticamente significativo (Tabla $N^{\circ} 4$ ).

\section{Tabla $N^{\circ} 1$}

Características generales de los niños evaluados

\begin{tabular}{lrc}
\hline & $\mathbf{n}$ & $\%$ \\
\hline Sexo & & \\
$\quad$ Masculino & 150 & 52.6 \\
$\quad$ Femenino & 135 & 47.4 \\
Grupo Etario & 52 & 18.2 \\
$\quad$ Lactante menor & 85 & 29.8 \\
$\quad$ Lactante mayor & 148 & 51.9 \\
$\quad$ Preescolar & & \\
Anemia & 113 & 39.6 \\
$\quad$ Si & 172 & 60.4 \\
$\quad$ No & & \\
Estado Nutricional & 59 & 20.7 \\
$\quad$ Desnutrido & 40 & 14.0 \\
$\quad$ Sobrepeso- obeso & 186 & 65.3 \\
$\quad$ Eutrófico & & \\
\hline
\end{tabular}


Para la realización de la segunda tabla se excluyeron a los pacientes con sobrepeso y obesidad, uniéndose las categorías de eutróficos con desnutridos leves y la de desnutridos moderados con los graves.

\section{Tabla $N^{\circ} 2$}

Pacientes anémicos y no anémicos según estado nutricional de los niños evaluados

\begin{tabular}{lrrrrr}
\cline { 2 - 6 } & \multicolumn{5}{c}{ Anemia } \\
\cline { 2 - 6 } & \multicolumn{3}{c}{ Si } & \multicolumn{1}{c}{ No } \\
\hline Estado Nutricional & & $\%$ & $n$ & $\%$ & Chi cuadrado (p) \\
$\quad$ Desnutridos moderados-graves & 6 & 6.0 & 2 & 1.4 & 4.000 \\
$\quad$ Eutróficos y Desnutridos leves & 94 & 94.0 & 143 & 98.6 & $(0.045)$ \\
\hline
\end{tabular}

Tabla $\mathrm{N}^{\circ} 3$

Anemia según sexo de los niños evaluados

\begin{tabular}{lrccccc}
\cline { 2 - 6 } & \multicolumn{5}{c}{ Anemia } \\
\cline { 2 - 6 } & \multicolumn{3}{c}{ Si } & \multicolumn{5}{c}{ No } \\
\hline Sexo & $\mathrm{n}$ & $\%$ & $\mathrm{n}$ & $\%$ & Chi cuadrado (p) \\
$\quad$ Masculino & 55 & $48.7 \%$ & 95 & $55.2 \%$ & 1.177 \\
$\quad$ Femenino & 58 & $51.3 \%$ & 77 & $44.8 \%$ & \\
\hline
\end{tabular}

Tabla $N^{\circ} 4$

Anemia según desnutrición de los niños evaluados

\begin{tabular}{lcccccc}
\cline { 2 - 6 } & \multicolumn{5}{c}{ Anemia } \\
\cline { 2 - 6 } & \multicolumn{3}{c}{ Si } & \multicolumn{4}{c}{ No } \\
\cline { 2 - 6 } Desnutrición & $\mathrm{n}$ & $\%$ & $\mathrm{n}$ & $\%$ & Chi cuadrado (p) \\
$\quad \mathrm{Si}$ & 27 & $23.9 \%$ & 32 & $18.6 \%$ & $(0.281)$ \\
$\mathrm{No}$ & 86 & $76.1 \%$ & 140 & $81.4 \%$ & \\
\hline
\end{tabular}

\section{DISCUSIÓN}

En lo que concierne a la prevalencia, las cifras en el Ecuador son equiparables a la de otros países donde a nivel nacional la prevalencia de anemia en infantes en etapa preescolar es de $25.7 \%$, si bien en otras regiones como México este dato es de $19.9 \%$ hay otros casos con valores más bajos como es Argentina con 7.6\%, Chile y Costa Rica con 4\% para ambos ${ }^{11}$, esta investigación arrojó una prevalencia mucho más alta a la conseguida en la bibliografía con un $39.6 \%$.

En este trabajo, al considerar la prevalencia de la anemia de acuerdo con el género, el femenino arrojó una frecuencia superior y es un dato que difiere al de un informe hecho anteriormente en 7513 infantes con edades comprendidas entre 2 y 5 meses evaluados en centros de salud públicos en Perú, en este caso las medianas de hemoglobina fueron similares 
en los dos sexos con $11.2 \mathrm{~g} / \mathrm{dl}$, las niñas (9.4\%) mostraron una prevalencia más baja respecto a su contraparte masculina $(10.9 \%)^{12}$.

En otro orden de ideas, en un análisis llevado a cabo en el Ecuador acerca de la prevalencia del sobrepeso y la obesidad se obtuvo que el $10 \%$ de los infantes tenía sobrepeso, mientras que un $3.6 \%$ obesidad $^{13}$, dicho esto, en el presente trabajo la prevalencia de ambas comorbilidades fue de $14 \%$, siendo excluidas del análisis estadístico debido a que pudieran sesgar los resultados.

De acuerdo con un análisis efectuado en una zona precaria de Uruguay que tomó en cuenta infantes del grupo etario de 6 a 48 meses de edad, consiguieron que la prevalencia de la obesidad y sobrepeso fue del $4.5 \%$, valor por debajo a lo alcanzado en el presente estudio ${ }^{14}$.

Seguidamente, en este trabajo se obtuvo que el 20.7\% de los infantes estaban desnutridos, cifra que al ser equiparada con las conseguidas en un estudio chileno el cual obtuvo que el $2.9 \%$ de los evaluados se encontraba en dicho estado nutricional, se entiende que esta condición permanece como un grave problema de salud pública ${ }^{15}$.

Conforme a un análisis efectuado en Perú en un grupo de indígenas con edades por debajo de los 5 años, se obtuvo una prevalencia de desnutrición crónica del 43\%, tomando en cuenta que estas poblaciones sufren de notorias deficiencias a nivel económico con escaso acceso a servicios de salud básico y conviven mayormente en estado de pobreza, siendo estos factores posibles agentes incidentes en la alta prevalencia de este estado nutricional ${ }^{16}$.
En el presente trabajo se determinó que el $39.6 \%$ de los estudiados tenía anemia, que al vincularlo con el estado nutricional se encontró una asociación estadísticamente significativa de acuerdo con los niveles de desnutrición, resultando que los niños anémicos mostraron una desnutrición de tipo moderada-grave. Esta información es similar a un informe de Brasil que tomó en cuenta infantes en el mismo grupo etario, consiguiendo una prevalencia de esta enfermedad del $56.6 \%$, igualmente siendo ligada de manera importante al bajo peso ${ }^{17}$.

Desde otro ángulo, una investigación efectuada en Perú en infantes entre 2 y 5 meses obtuvo que la desnutrición crónica se posicionó como un agente relacionado a la anemia, gracias al modelo multivariante ajustado por región, sexo y altitud con un $\mathrm{OR}=1.3$; IC 95\%=1.01.6; $p=0.025$, por su parte, la obesidad se ubicó como un elemento protector para esta enfermedad de orden hematológico ${ }^{18}$.

En concordancia con los resultados de este estudio, la sobrenutrición no arrojó un vínculo significativo con el diagnóstico de anemia por lo cual estos pacientes se excluyeron para evitar sesgos al momento de evaluar el estado nutricional y la anemia, esta información es parecida a la de una investigación efectuada en Lima, Perú en 2286 niños y adolescentes, donde el 20.7\% tenía menos de 5 años y obteniendo medias de hemoglobina y elevada prevalencia de esta enfermedad respecto a todas las edades por encima de los 5 años ${ }^{19}$. Además, otro análisis llevado a cabo en Tayikistán que consideró 1342 infantes con un rango etario de 25 a 60 meses consiguió que la prevalencia de sobrepeso fue del 13\% y un 9.5\% para la obesidad, siendo esta última cifra superior a la del presente trabajo; sin embargo, no obtuvieron una relación significativa entre la anemia y la obesidad ${ }^{20}$. 


\section{CONCLUSIONES}

Existe una relación significativa entre la anemia y los grados de desnutrición leve y moderada; se destaca la alta prevalencia de anemia de predominio ligero en el sexo femenino. El ser eutrófico no es un criterio de exclusión para la presencia de anemia, por lo cual es imperante tomar en cuenta otros factores asociados, como comorbilidades o antecedentes que no se pueden dejar pasar por alto al momento de la evaluación de un lactante o preescolar, con la finalidad de disminuir la morbimortalidad asociada a la anemia tanto en sus episodios agudos como crónicos.

\section{ASPECTOS BIOÉTICOS}

La investigación fue aprobada por el Consejo Directivo del Departamento de Posgrados de la Universidad del Azuay y posteriormente se obtuvo la autorización por parte del Director Médico del Hospital Pablo Jaramillo Crespo.

\section{INFORMACIÓN DEL AUTOR}

Blacio Vidal Willie Jack. Médico. Especialista en Pediatría. Hospital Humanitario Pablo Jaramillo. Unidad de Pediatría. Cuenca - Azuay - Ecuador.

e-mail: wilblavi@hotmail.com

ORCID:https://orcid.org/0000-0002-8494-9429

\section{CONFLICTO DE INTERESES}

El autor no reporta conflicto de intereses.

\section{FUENTES DE FINANCIAMIENTO}

Fondos propios.

\section{REFERENCIAS BIBLIOGRÁFICAS}

1. ENSANUT. ENSANUT-ECU 2012. Ministerio de Salud Pública/Instituto Nacional de Estadísticas y Censos. Quito-Ecuador. 2014 [citado 8 de septiembre de 2018]; Disponible en: http://www.ecuadorencifras.gob.ec/ documentos/web-inec/Estadisticas_ Sociales/ENSANUT/MSP_ENSANUTECU_06-10-2014.pdf

2. Urquidi B, Vera A, Trujillo B, Mejía S. Prevalencia de anemia en niños de 6 a 24 meses de edad de tres centros de salud de la ciudad de La Paz. Rev Soc Boliv Pediatría [Internet]. agosto de 2006 [citado 8 de septiembrede2018];45(3):153-6.Disponible en: $\quad$ http://www.scielo.org.bo/scielo. php? script $=$ sci_abstract $\&$ pid $=S 1024$ 06752006000300003\&lng=es\&nrm=iso\&t| $\mathrm{ng}=\mathrm{es}$

3. WHO. Haemoglobin concentrations for the diagnosis of anaemia and assessment of severity [Internet]. 2011 [citado 7 de septiembre de 2018]. Disponible en: http://www.who.int/vmnis/indicators/ haemoglobin.pdf

4. De Benoist B. Worldwide prevalence of anaemia 1993-2005 of: WHO Global Database of anaemia [Internet]; 2008 [citado 8 de septiembre de 2018]. Disponible en: http://whqlibdoc.who.int/ publications/2008/9789241596657_eng. pdf

5. OMS. OMS | Datos y cifras sobre obesidad infantil [Internet]. WHO. 2018 [citado 3 de octubre de 2018]. Disponible en: http:// www.who.int/end-childhood-obesity/ facts/es/

6. Tsui AKY, Marsden PA, Mazer CD, Sled JG, Lee KM, Henkelman RM, et al. Differential HIF and NOS responses to acute anemia: defining organ-specific hemoglobin thresholds for tissue hypoxia. Am J PhysiolRegul Integr Comp Physiol [Internet]. julio de 2014 [citado 8 de septiembre de 2018];307(1):13-25. Disponible en: http://www.physiology.org/doi/10.1152/ ajpregu.00411.2013 
7. Qari MH, Wali Y, Albagshi MH, Alshahrani M, Alzahrani A, Alhijji IA, et al. Regional consensus opinion for the management of Beta thalassemia major in the Arabian Gulf area. Orphanet J Rare Dis [Internet]. 17 de septiembre de 2013 [citado 8 de septiembre de 2018];8(1):143-7. Disponible en: https://doi.org/10.1186/1750-1172-8143

8. Adjagba PM, Habib G, Robitaille N, Pastore Y, Raboisson M-J, Curnier D, et al. Impact of sickle cell anaemia on cardiac chamber size in the paediatric population. Cardiol Young. julio de 2017;27(5):918-24.

9. Wang J, Chang S, Zhao L, Yu W, Zhang J, Man Q, et al. Effectiveness of communitybased complementary food supplement (Yingyangbao) distribution in children aged 6-23 months in poor areas in China. PLOS ONE [Internet]. 20 de marzo de 2017 [citado 7 de septiembre de 2018];12(3):1-8. Disponible en: http://dx.plos.org/10.1371/ journal.pone.0174302

10. Tulchinsky TH. Micronutrient Deficiency Conditions: Global Health Issues. Public Health Rev [Internet]. 1 de junio de 2010 [citado 7 de septiembre de 2018];32(1):24355. Disponible en: https://doi.org/10.1007/ BF03391600

11. Ramírez-Vélez R, Matínez-Torres J, Meneses-Echavez JF. Prevalencia y factores sociodemográficos asociados a la deficiencia de ferritina en niños de Colombia, 2010. Rev Peru Med Exp Salud Pública [Internet]. junio de 2014 [citado 8 de septiembre de 2018];31:237-42. Disponible en: https://www.scielosp.org/ scielo. php?script=sci_arttext\&pid=S172646342014000200007\&lng=en
12. Álvarez-Dongo D, Sánchez-Abanto J, Gómez-Guizado G, Tarqui-Mamani C. Sobrepeso y obesidad: prevalencia y determinantes sociales del exceso de peso en la población peruana (2009-2010). Rev Peru Med Exp Salud Pública [Internet]. septiembre de 2012 [citado 3 de junio de 2018];29:303-13. Disponible en: https:// www.scielosp.org/article/rpmesp/2012. v29n3/303-313/

13. Oleas G M. Prevalencia y factores de riesgo de sobrepeso y obesidad en escolares de la provincia de Imbabura: Ecuador. 2010. Rev Chil Nutr [Internet]. marzo de 2014 [citado 8 de septiembre de 2018];41(1):61-6. Disponible en: $\quad$ https://scielo.conicyt.cl/scielo. php?script =sci_abstract $\&$ pid $=$ S0717$75182014000100008 \&$ lng =es\&nrm=iso\&t| $\mathrm{ng}=\mathrm{es}$

14. Assandri E, Skapino E, Da Rosa D, Alemán A, Acuña AM, Assandri E, et al. Anemia, estado nutricional y parasitosis intestinales en niños pertenecientes a hogares vulnerables de Montevideo. Arch Pediatría Urug [Internet]. abril de 2018 [citado 31 de julio de 2020];89(2):86-98. Disponible en: $\quad$ http://www.scielo.edu.uy/scielo. php? script $=$ sci_abstract $\&$ pid $=$ S1688 12492018000200086\&lng=es\&nrm=iso\&tl $\mathrm{ng}=\mathrm{es}$

15. Bustos $P$, Muñoz S, Vargas $C$, Amigo $\mathrm{H}$. Pobreza y procedencia indígena como factores de riesgo de problemas nutricionales de los niños que ingresan a la escuela. Salud Pública México [Internet]. junio de 2009 [citado 8 de septiembre de 2018];51(3):187-93. Disponible en: http://www.scielosp.org/ scielo. php?script=sci_arttext\&pid=S003636342009000300008\&lng=es\&nrm=iso\&t| ng=es 
16. Bendezú G, Espinoza D, Bendezú-Quispe G, Torres-Román JS, Huamán-Gutiérrez RM. Características y riesgos de gestantes adolescentes. Rev Peru Ginecol Obstet [Internet]. enero de 2016 [citado 6 de julio de 2020];62(1):13-8. Disponible en: $\quad$ http://www.scielo.org.pe/scielo. php?script $=$ sci_abstract $\&$ pid $=$ S2304$51322016000100002 \& \mathrm{lng}=e s \& n r m=i s o \& t$ ng=es

17. Santos RF dos, Gonzalez ESC, Albuquerque EC de, Arruda IKG de, Diniz A da S, Figueroa $J N$, et al. Prevalence of anemia in under five-year-old children in a children's hospital in Recife, Brazil. Rev Bras Hematol E Hemoter [Internet]. 2010 [citado 8 de septiembre de 2018];33(2):1004. Disponible en: http://www.rbhh. org/?doi=10.5581/1516-8484.20110028

18. Gómez-Guizado G, Munares-García O. Anemia y estado nutricional en lactantes de dos a cinco meses atendidos en establecimientos del Ministerio de Salud del Perú, 2012. Rev Peru Med Exp Salud Publica [Internet]. julio de 2014 [citado 31 de julio de 2020];31(3):487-93. Disponible en: $\quad$ http://www.scielo.org.pe/scielo. php?script=sci_abstract $\&$ pid $=$ S1726$46342014000300012 \&$ lng=es\&nrm=iso\&t| $\mathrm{ng}=\mathrm{es}$

19. Rodríguez-Zúñiga MJ. Obesidad, sobrepeso y anemia en niños de una zona rural de lima, Perú. Medicina (Mex). 2015;75(1):379-83.

20. Crivelli M, Wyss K, Grize L, Matthys B, Aebi T, Zemp E. Are overweight and obesity in children risk factors for anemia in early childhood? Results from a national nutrition survey in Tajikistan. Int J Public Health. 19 de marzo de 2018;63. 